\title{
Research on Denoising Methods in Power Line Communication
}

\author{
Hongyan Yin \\ Beijing Inspection and Quarantine Testing Center of \\ Beijing Entry-Exit Inspection and Quarantine Bureau \\ 100026 China \\ Yujun Liu \\ Beijing Inspection and Quarantine Testing Center of \\ Beijing Entry-Exit Inspection and Quarantine Bureau \\ 100026 China
}

\begin{abstract}
With the development of power line communication (PLC) technology, it is widely used in smart grid. In this paper, we mainly introduce communication modes of PLC, and respectively analysis their advantages and disadvantages. Moreover channel noises are described in the paper, and we respectively simulate background and impulsive noise in modeling. Finally we introduce compressed sensing and Sparse Bayesian Learning denoising methods. Using MATLAB to simulate them, we analyze the simulation result and compare their signal to noise ratio (SNR).
\end{abstract}

Keywords- power line communicatio; background noise; impulsive noise; $C S ; S B L$.

\section{INTRODUCTION}

Power lines are used as medium to transmit in PLC. Power line is also an existing infrastructure. It is only used in remote meter reading and home furnishing automation. Its transmission rate is very low, and it isn't suited to transfer high speed information. With the rapid development of network technique information technology, high speed power line carrier technology that used low voltage power line to transfer $1 \mathrm{Mbps}$ information. This technology can realize multi-service carrying, such as data, voice and video[1]. With the continuous improvement of high speed PLC technology, it can realize four networks unifying that can transfer data, voice, video and electric power. This technology is seductive and economical. Moreover, because PLC technology has the advantages that simple networking, low cost, high reliability and easy realization, it has attracted more and more attentions. The network block diagram of PLC system is shown in Fig .1.

\author{
Tong $\mathrm{Wu}$ \\ China National Institute of Metrology
}

\section{Power Line Communication Modes}

The function block diagram of PLC is shown in Fig .2. In the Fig .2, $\mathrm{A}$ is a power station, and $\mathrm{B}$ is a power substation. $50 \mathrm{~Hz}$ current that power station generate is boosted, then it is sent to power substation through power line, and it is supplied to user after reduction voltage. The key problem is how to couple high-frequency signal into power line, when we use power line to realize PLC.

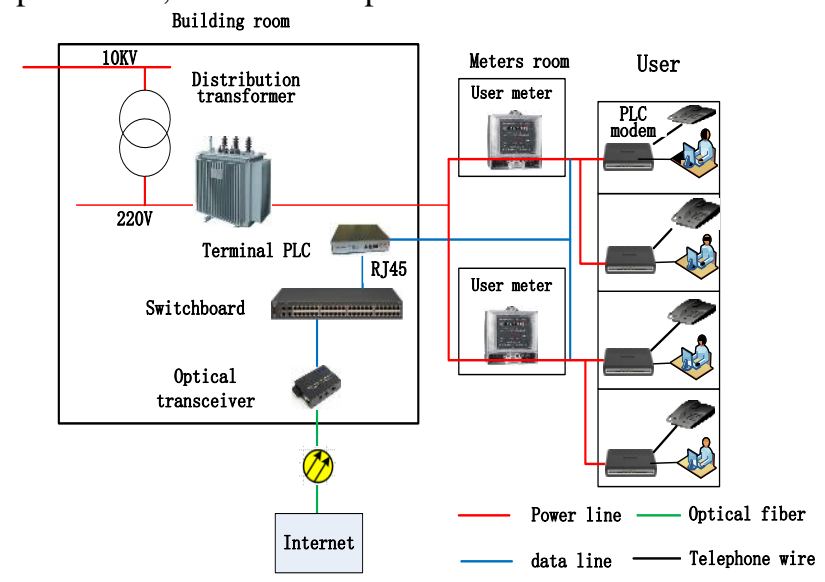

Figure 1. Network block diagram of PLC system

The key problem is how to couple high-frequency signal into power line, when we use power line to realize PLC. At present, PLC has the following several ways: 


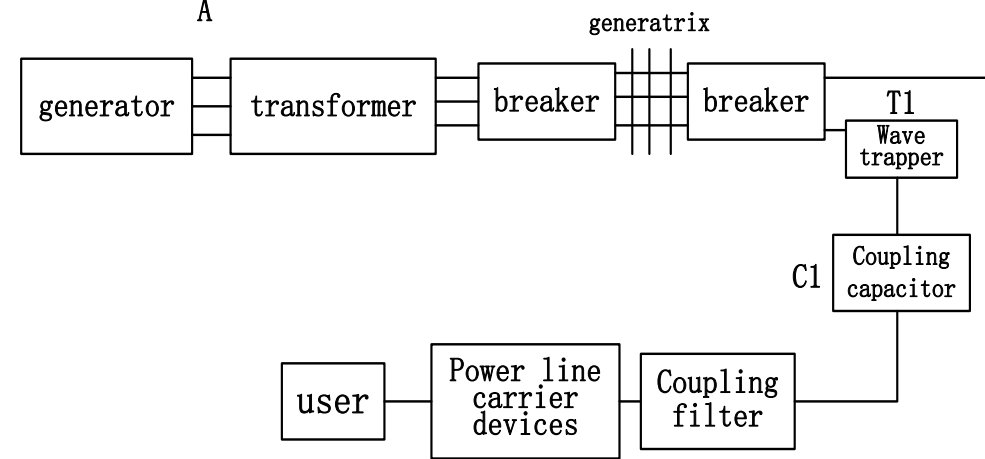

F1

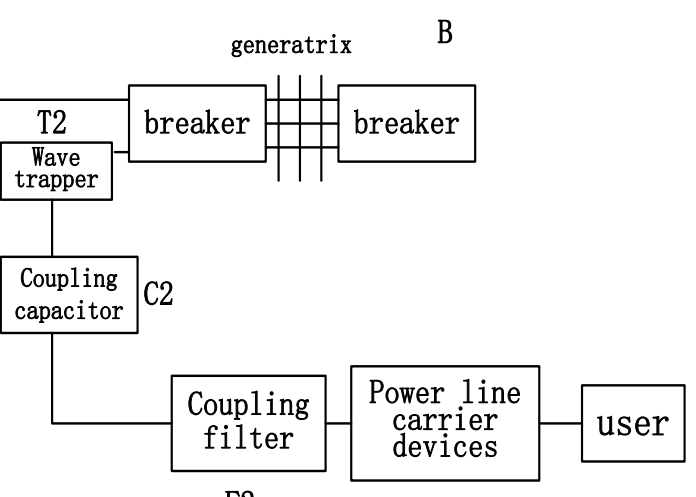

F2

Figure 2. The principle diagram of PLC

\section{A. Low-voltage power line communication (LPLC)}

LPLC is widely regarded as an important digital communication way that can replace private network in building automation, security monitoring, office automation and remote meter reading [2,3].

Because load is complex, various and random, and noise is diverse and strong in low-voltage network. This causes signal reflecting, resonance and standing wave. Moreover it leads to signal attenuation inordinately, and the channel of PLC has strong frequency selectivity.

Furthermore channel is time-varying, because lowvoltage power line is uneven for carrier signal and different loads are accessed or disconnected randomly in optional position of low-voltage network. In the meantime, there is large-scale line attenuation and impedance, so we need to improve analog front end technology. For example, we should do more researches about adaptive filtering and adaptive equalization.

\section{B. The medium of middle-voltage power line communication (MPLC)}

MPLC can be divided into overhead line and underground cable line. Moreover the theories that they establish carrier channel are different [4,5].

The first one can realize carrier signal coupling into overhead line through combining filter with high voltage isolation capacitance. Though the coupling efficiency of this carrier channel signal is fine, it is easily affected by circuit power supply environment. So the signal attenuation in line is instability, and the power supply mode will be changed.

The main carrier of signal is cable shielded layer in second mode. It is almost not affected by variable circuit power supply condition, and cable shielded layer is also not affected by variable power supply mode. Moreover it only dependents on topological relation of power supply, and it is the most ideal carrier signal medium. Coupling equipments can be installed conveniently, and they don't directly connect with high-voltage electrical devices.

\section{High-voltage power line communication (HPLC)}

HPLC uses high voltage transmission lines as communication channel. It has many advantages, such as small investment, fast benefit, high reliability and synchronization with power grid construction. So it is a main communication way of Chinese high voltage electricity system. However, due to the wicked channel condition and complex communication environment of high voltage power line, it makes realizing high-speed HPLC difficult[6].

We usually consider power frequency harmonic and radio transmitting interference in high voltage grid. Impedance of high voltage power line is generally $300 \sim 400 \Omega$, and it is fluctuant on line. According to field measurement, it will severely affect on carrier channel attenuation when fluctuation range is about $1 / 2$. Because carrier frequency band is limited to $40 \sim 500 \mathrm{kHz}$, as long as we control the harmonic of carrier equipment and transmitted power is very small, external interference will be avoided.

\section{NOISE OF PLC SYSTEM}

\section{A. Channel noise analysis}

The noise of PLC channel can be divided into several kinds shown in Fig .3.

1) Noise that has smooth spectral;

2) Narrow band noise;

3) Period noise is synchronous with power frequency;

4) Single event impulse noise has nothing to do with system frequency.

For the above noises, the variation of 1) is small, so it can be regarded as background noise; 3) and 4) are timevarying in $\mu \mathrm{s}$ or ms. When these pulses are generated, power spectrum of noise is high. So it is able to cause digit or string burst error during data transmitting.

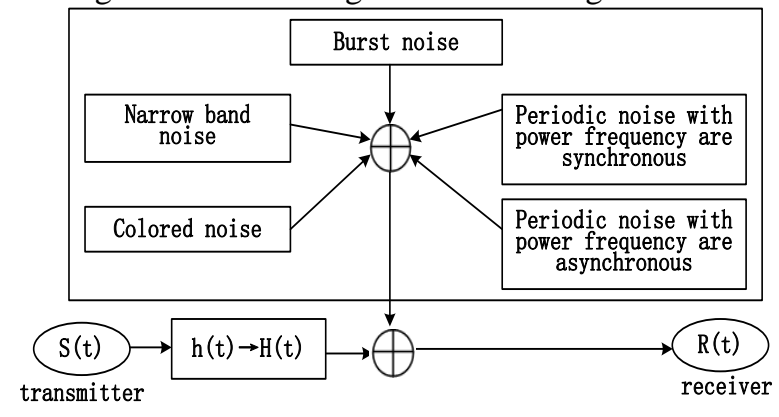

Figure 3. Noise classification of power line 


\section{B. Channel noise model}

If we establish modular according to basic classification of noise, any complex noise is able to be expressed by the sum of basic modules ${ }^{[7-9]}$.

\section{1) Background noise model}

Background can be generated by white noise source with filter according to Fig .3. This modeling method is widely accepted at present. Noise filtering transfer function $\mathrm{H}(\mathrm{z})$ in $\mathrm{z}$ plane can be described as following:

$$
H(z)=\frac{B(z)}{A(z)}=\frac{1+\sum_{i=1}^{m} b_{i} z^{-i}}{1+\sum_{j=1}^{n} a_{j} z^{-j}}
$$

Where $\mathrm{B}(\mathrm{z})$ denotes moving average portion, and $\mathrm{A}(\mathrm{z})$ denotes auto-regressive portion. Module parameters are made up of variance $\sigma^{2}$ of noise source and filter coefficients. Using auto-regressive to process model, $\mathrm{B}(\mathrm{z})=1$, parameters can be confirmed by noise signal measuring with auto-regressive frequency analyser.

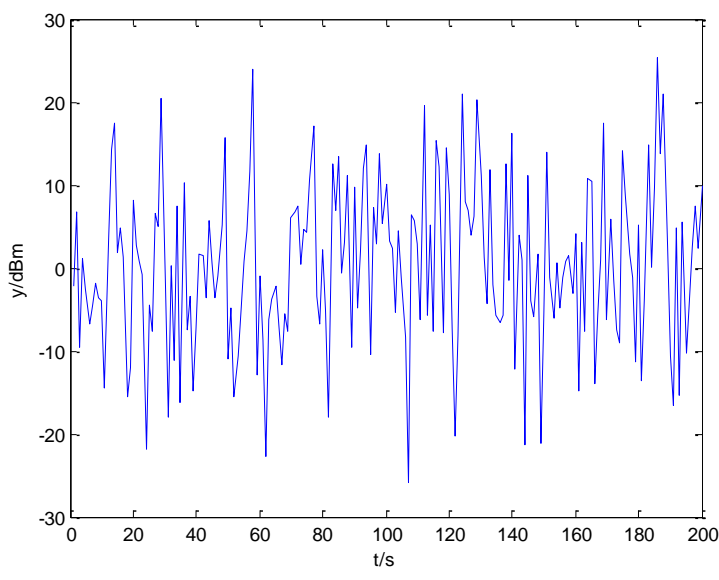

Figure 4. Background noise

\section{2) Impulsive noise model}

Impulsive noise can be divided into periodic impulsive noise and asynchronous impulsive noise. For the former, it is easily modeled because of its certainty. But the latter must be described by a random model, because the parameters of time and envelope are random. We usually use Gaussian mixture model to model asynchronous. A random variable $\mathrm{Z}$ is a Gaussian mixture distribution if its probability density function (pdf) is a weighted summation of different Gaussian distribution. It is as following.

$$
\mathrm{f}_{\mathrm{z}}(\mathrm{z})=\sum_{\mathrm{k}=0}^{\mathrm{K}} \pi_{\mathrm{k}} \cdot \mathrm{N}\left(\mathrm{z} ; 0 ; \gamma_{\mathrm{k}}\right)
$$

Where $N\left(z ; 0 ; \gamma_{k}\right)$ is a Gaussian pdf with zero mean and $\gamma_{\mathrm{k}}$ variance. $\pi_{\mathrm{k}}$ is the mixing probability of the $\mathrm{k}$-th Gaussian component.

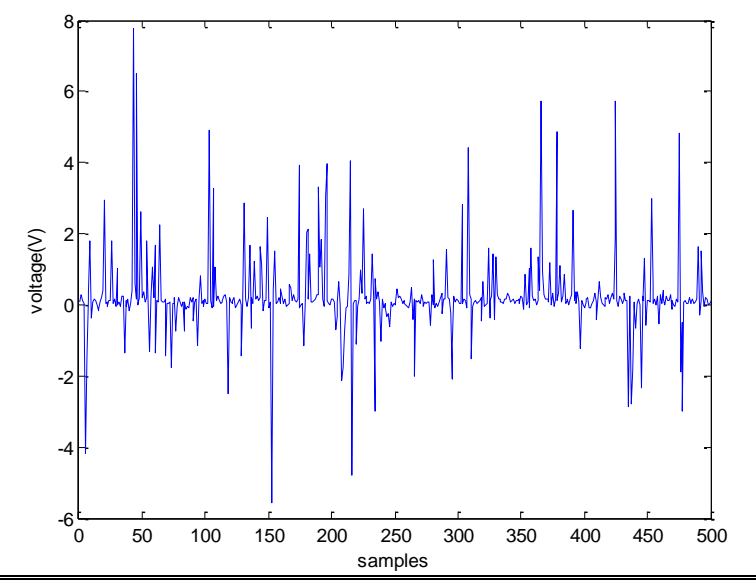

Figure 5. Asynchronous impulsive noise generated by a Gaussian mixture distribution with $\pi_{\mathrm{k}}=[0.7,0.16,0.14]$ and $\gamma_{\mathrm{k}}=[1,100,1000]$.

\section{MAIN DENOISING Methods of PLC System}

\section{A. Compressed Sensing $(C S)$}

CS is based on signal sparse character. It can realize signal reconstruction in the sampling frequency that is less than Nyquist. This largely reduces the cost of signal processing. The principle of compressed sensing is as following.

The transformation coefficient vector of signal $f \in \mathrm{R}^{\mathrm{N}}$ based on $\Psi$ is $\mathrm{x}=\Psi^{-1} \mathrm{f}$. $\mathrm{x}$ is sparse, namely it has few nonzero value. Measurement matrix is designed to acquire projection of signal $\mathrm{f}$,

$$
\mathrm{y}=\Phi f=\Phi \Psi \mathrm{x}=\mathrm{Ax}, \mathrm{y} \in \mathrm{R}^{\mathrm{M}}
$$

Where $\Phi$ is measurement matrix, $\Psi$ is sparse base, and $\mathrm{A}$ is compressed sensing.

We can recover original signal $f$ from $y$ by specific reconstruction algorithm.

\section{B. Sparse Bayesian Learning $(S B L)^{[10]}$}

SBL is generally used to solve the linear regression problem.

$$
y=A x+w, w \sim C N\left(0, \sigma^{2} I_{M}\right)
$$

Where $\mathrm{y} \in \mathrm{C}^{\mathrm{M}}$ is an observation vector, $\mathrm{A}=$ $\left[\mathrm{A}_{1}, \mathrm{~A}_{2}, \ldots, \mathrm{A}_{\mathrm{N}}\right] \in \mathrm{C}^{\mathrm{M} \times \mathrm{N}}$ is an overcomplete basis, and $\mathrm{x} \in \mathrm{C}^{\mathrm{N}}$ is a sparse weight vector to be estimated.

SBL imposes a parameterized Gaussian prior on $\mathrm{x}$

$$
\mathrm{p}(\mathrm{x} ; \mathrm{T})=\mathrm{CN}(\mathrm{w} ; 0 ; \mathrm{T})
$$

WhereT $\triangleq \operatorname{diag}\{\gamma\}$, and $\gamma \in \mathrm{R}^{\mathrm{N}}$ whose $\mathrm{j}$-th component $\gamma_{j}$ is the variance of $x_{j}$. Given the prior, the likelihood of the observation can be expressed as

$$
\mathrm{p}\left(\mathrm{y} ; \mathrm{T} ; \sigma^{2}\right)=\mathrm{CN}\left(\mathrm{t} ; 0 ; \mathrm{ATA}^{*}+\sigma^{2} \mathrm{I}_{\mathrm{M}}\right)
$$

A maximum likelihood (ML) estimator is used to solve the hyperparameters $\gamma$ and $\sigma^{2}$ that maximize (6). The ML estimation is computed iteratively using expectation maximization (EM). 
Given the observations and the estimated hyperparameters, the posterior density of $\mathrm{x}$ is also a Gaussian distribution

$$
\begin{aligned}
& \mathrm{p}\left(\mathrm{x} \mid \mathrm{y} ; \mathrm{T}, \sigma^{2}\right)=\mathrm{CN}\left(\mathrm{x} ; \mu_{\mathrm{x}} ; \sum_{\mathrm{x}}\right) \\
& \mu_{\mathrm{x}}=\sigma^{-2} \sum_{\mathrm{x}} \mathrm{A}^{*} \mathrm{y} \\
& \sum_{\mathrm{x}}=\left(\sigma^{-2} \mathrm{~A}^{*} \mathrm{~A}+\mathrm{T}^{-1}\right)^{-1}
\end{aligned}
$$

The maximum a posteriori (MAP) estimate of $\mathrm{x}$ is the posterior mean $\mu_{\mathrm{x}}$.

Fig .6 is the simulation result of noise mitigation used $\mathrm{CS}$ and SBL respectively. We can obviously observe that SNR of SBL than CS is increased, when the BER is same.

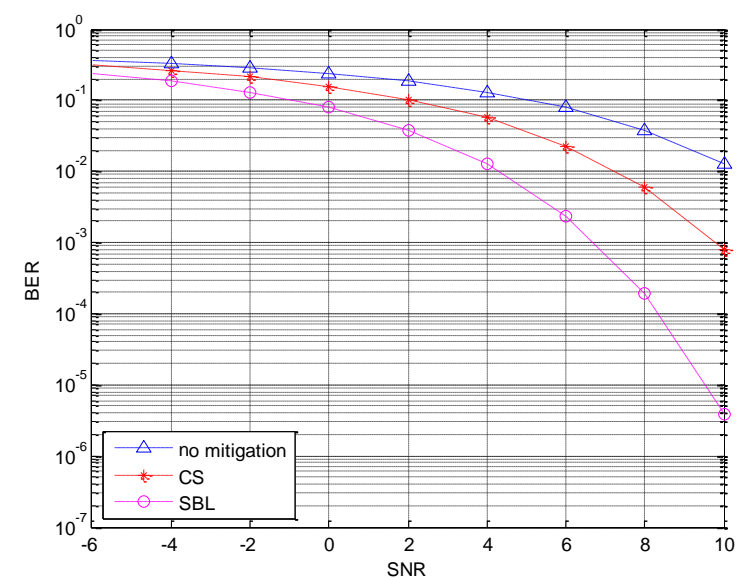

Figure 6. BER performance of the CS and SBL for impulsive noise in Gaussian mixture

\section{CONCLUSION}

This paper uses CS and SBL to mitigate noise in the PLC system. Through observing and analyzing simulation result, CS and SBL not only can effectively mitigate noise and enhance original signal, but also can improve reliability and ensure system communication safe. They also can increase SNR. All of this keep PLC technology more popular.

\section{ACKNOWLEDGMENT}

This work is supported by the national quality inspection service project under Grant 201410050.

The PLC signals is pre-procssed by the software titled "SmartSignalProcessing" developed by Prof. Ming-Yue Zhai at North China Electric Power University.

\section{REFERENCES}

[1] Janse van Rensburg P.A , Sibanda M.P , Ferreira H.C . Integrated Impedance-Matching Coupler for Smart Building and Other Power-Line Communications Applications[J] . IEEE Transactions on Power Delivery,2015,30(2):949-956.

[2] Matanza J, Alexandres S, Rodríguez-Morcillo C. Advanced metering infrastructure performance using European low-voltage power line communication networks[J]. IEEE Transactions on Communications, 2014,8(7):1041-1047.

[3] GUO Haokun, WU Junji, HENG Sikun, et al. Modeling and simulation of the noise in low voltage power line communication channel[J] . Power Syetem Protection and Control,2012, 40(13):61-66

[4] Milioudis A.N , Syranidis K.N , Andreou G.T , Labridis D.P. Modeling of Medium-Voltage Power-Line Communication Systems Noise Levels[J] . IEEE Transactions on Power Delivery,2013,28(4):2004-2013.

[5] Artale G, Cataliotti A, Cosentino V, Di Cara D, Fiorelli R, Russotto P, Tine G. Medium Voltage Smart Grid: Experimental Analysis of Secondary Substation Narrow Band Power Line Communication $[\mathrm{J}]$. IEEE Transactions on Instrumentation and Measurement,2013, 62(9):2391-2398.

[6] Yin Kang. Study on self-adaptive OFDM technology of highvoltage power line carrier communication[C] . Chongqing University,2006.

[7] Guzel T, Ustunel E, Celebi H.B, Deliç H, Mihcak K. Noise Modeling and OFDM Receiver Design in Power-Line Communication[J] . IEEE Transactions on Power Delivery, 2011,26(4):2735-2742.

[8] WU Jun-ji, GUO Hao-kun, MENG Shao-liang, YING Zhaofeng. Modeling of background noise in power line communication channel[J]. Power System Protection and Control, 2011, 39(23):610 .

[9] GUO Haokun, WU Junji, YING Zhanfeng, et al. Application of improved Markov chain in impulse noise modeling of power line communication channel[J]. Power System Protection and Control,2012,40(5):129-132.

[10] LIN J, NASSAR M, EVANS B L. Impulsive noise mitigation in power line communication using sparse Bayesian learning $[\mathrm{J}]$. IEEE Journal on Selected Areas in Communications, 2013, 31(7):1172-1183. 